\title{
NeuroImage
}

ELSEVIER

www.elsevier.com/locate/ynimg

NeuroImage 32 (2006) $1158-1166$

\section{Multifractal analysis of deep white matter microstructural changes on MRI in relation to early-stage atherosclerosis}

\author{
Tetsuya Takahashi, ${ }^{\text {a }}$ Tetsuhito Murata, ${ }^{\mathrm{a}, *}$ Kosuke Narita, ${ }^{\mathrm{a}}$ Toshihiko Hamada, ${ }^{\mathrm{b}}$ \\ Hirotaka Kosaka, ${ }^{\mathrm{a}}$ Masao Omori, ${ }^{\mathrm{a}}$ Koichi Takahashi, ${ }^{\mathrm{c}}$ Hirohiko Kimura, ${ }^{\mathrm{d}}$ \\ Haruyoshi Yoshida, ${ }^{\mathrm{b}}$ and Yuji Wada ${ }^{\mathrm{a}}$ \\ ${ }^{a}$ Department of Neuropsychiatry, Faculty of Medical Sciences, University of Fukui, 23-3 Shimoaizuki, Matsuoka-cho, Yoshida-gun, Fukui 910-1193, Japan \\ ${ }^{\mathrm{b}}$ Department of Clinical and Laboratory Science, Faculty of Medical Sciences, University of Fukui, Fukui 910-1193, Japan \\ ${ }^{\mathrm{c}}$ Department of Informatics, Faculty of Science and Engineering, Kinki University, Higashi-Osaka, Japan \\ ${ }^{\mathrm{d}}$ Department of Radiology, Faculty of Medical Sciences, University of Fukui, Fukui 910-1193, Japan
}

Received 26 September 2005; revised 18 April 2006; accepted 28 April 2006

Available online 11 July 2006

\begin{abstract}
Multifractal analysis based on generalized concepts of fractals has been applied to evaluate biological tissues composed of complex structures. This type of analysis can provide a precise quantitative description of a broad range of heterogeneous phenomena. Previously, we applied multifractal analysis to describe heterogeneity in white matter signal fluctuation on T2weighted MR images as a new method of texture analysis and established $\Delta \alpha$ as the most suitable index for evaluating white matter structural complexity (Takahashi et al. J. Neurol. Sci., 2004; 225: 33-37). Considerable evidence suggests that pathophysiological processes occurring in deep white matter regions may be partly responsible for cognitive deterioration and dementia in elderly subjects. We carried out a multifractal analysis in a group of 36 healthy elderly subjects who showed no evidence of atherosclerotic risk factors to examine the microstructural changes of the deep white matter on T2-weighted MR images. We also performed conventional texture analysis, i.e., determined the standard deviation of signal intensity divided by mean signal intensity (SD/MSI) for comparison with multifractal analysis. Next, we examined the association between the findings of these two types of texture analysis and the ultrasonographically measured intima-media thickness (IMT) of the carotid arteries, a reliable indicator of early carotid atherosclerosis. The severity of carotid IMT was positively associated with $\Delta \alpha$ in the deep white matter region. In addition, this association remained significant after excluding 12 subjects with visually detectable deep white matter hyperintensities on MR images. However, there was no significant association between the severity of carotid IMT and SD/MSI. These results indicate the potential usefulness of applying multifractal analysis to conventional MR images as a new approach to detect the microstructural changes of apparently normal white matter during the early stages of atherosclerosis.

(C) 2006 Elsevier Inc. All rights reserved.
\end{abstract}

Keywords: Texture analysis; Multifractal; T2-weighted MRI; Deep white matter; Intima-media thickness; Atherosclerosis

\footnotetext{
* Corresponding author. Fax: +81 776618136 .

E-mail address: tmurata@fmsrsa.fukui-med.ac.jp (T. Murata).

Available online on ScienceDirect (www.sciencedirect.com).
}

\section{Introduction}

Considerable evidence has recently suggested that pathophysiological processes in the white matter regions may partly account for a number of brain dysfunctions in elderly subjects. This white matter deterioration may play an important role in clinical deficits such as age-related cognitive decline, geriatric depression, or dementia (Gunning-Dixon and Raz, 2003; Valenzuela et al., 2000). Therefore, the deterioration of white matter should be evaluated at the earliest possible stage. T2-weighted MRI has demonstrated a high prevalence of white matter changes with aging. These changes frequently occur in deep white matter, i.e., the terminal zone of the penetrating medullary arteries (Kirkpatrick and Hayman, 1987; van Swieten et al., 1991), and they are reflected in MR signals such as deep white matter hyperintensities (DWMHs). DWMHs are related to standard risk factors for cardiovascular diseases and are believed to be a consequence of cerebrovascular disease (Bots et al., 1993; Fazekas et al., 1988; Manolio et al., 1994).

Another method, ultrasonographic measurement of the intimamedia thickness (IMT) of carotid arteries, is reported to be a reliable indicator of the early stage of atherosclerosis (Allan et al., 1997; Burke et al., 1995). Given that the carotid arteries provide most of the blood supply to the brain, carotid atherosclerotic changes of IMT may be associated with a higher risk of cerebrovascular disease (Manolio et al., 1999; Touboul et al., 2000) and are regarded as a robust predictor of stroke (Cao et al., 2003; O'Leary et al., 1999). Several studies have also elucidated the relationship between IMT and visible white matter hyperintensities on MRI (Manolio et al., 1999; Pico et al., 2002).

The analysis of texture parameters is a useful way of increasing the information obtainable from MR signal changes. Various types of texture analysis have been applied for the delineation and separation of tissue alterations (Castellano et al., 2004). However, the current clinical diagnosis of white matter degeneration on T2weighted MR images still depends on visual rating scales due to 
the lack of appropriate measurement tools. Accordingly, there is demand for a more sophisticated method that can provide a better description of pathological changes on MR images, especially in the early stage. Fractals, proposed by Mandelbrot (1983), are characterized by self-similar structures, and fractal theory provides a new scale of nonlinearity. Various physical phenomena such as the growth of cancers and arterial and bronchial trees show the features of fractals, and fractal theory has already provided clinically useful information to differentiate pathological tissue from healthy tissue (Baish and Jain, 2000; Mauroy et al., 2004; Zamir, 1999). The application of fractal theory to the neuroimaging field has also provided novel and useful information in clinical medicine (Kiselev et al., 2003; Yoshikawa et al., 2003a,b). In contrast to simple fractals, the concept of "multifractal phenomena" holds that different regions of an object have different local fractal properties that quantify local singular behavior (Halsey et al., 1986). To describe these local fractal properties, Halsey et al. (1986) introduced $\alpha$, the so-called Lipschitz-Hölder index (or the strength of singularity), and the multifractal spectrum $f(\alpha)$. Multifractal scaling provides a quantitative description of a broad range of heterogeneous complex phenomena that are impossible to evaluate using simple fractals (Ivanov et al., 1999; Shimizu et al., 2004; Stanley et al., 1999).

Our previous study showed that index $\Delta \alpha$ (established as the most suitable index of heterogeneity in Takahashi et al. (2001)), in apparently normal deep white matter frontal regions on T2-MRI, reached significantly higher levels in elderly subjects than in young subjects and was correlated with cognitive decline in elderly subjects (Takahashi et al., 2004). Yet, the underlying pathogenesis behind these changes in the deep white matter in elderly subjects remains obscure. In this study, we carried out a multifractal analysis to examine the microstructural changes of the deep white matter in apparently normal T2-weighted MR images in elderly subjects who showed no evidence of atherosclerotic risk factors. To clarify the utility of multifractal analysis, we additionally calculated the standard deviation of signal intensity divided by the mean signal intensity (SD/MSI) as a conventional type of texture analysis in comparison with multifractal analysis. We also sought to identify the pathogenesis of these changes with special reference to ultrasonographic measurements of carotid artery IMT, a reliable indicator of the early stage of atherosclerosis. Last, we re-examined the relationships among these changes in view of the coexistence of DWMH.

\section{Materials and methods}

\section{Subjects and study design}

Forty-three healthy elderly subjects were recruited from the general population in Fukui prefecture, Japan; a brochure described the following exclusion criteria: history of major atherosclerotic risk factors (such as hypertension, hypercholesterolemia, diabetes mellitus, coronary artery disease, congestive heart failure, and hemodynamically significant valvular disease), history of psychiatric treatment, chronic alcoholism, smoking, obesity with a body mass index (BMI) above 26, and the continuous administration of drugs. All female subjects were postmenopausal. Of the 43 volunteers, 7 were excluded due to the confined space for setting the ROI and a high prevalence of DWMH, which precluded subsequent multifractal analysis (as described in the Multifractal analysis section). Finally, 36 volunteers (23 males aged 52-72 (mean: $61.0 \pm 4.7$ years) and 13 females aged 55-70 (mean: $60.9 \pm$ 4.1 years)) were included in the study. All subjects gave written informed consent.

The protocol of this study was approved by the ethics committee of our university. Data from each examination were analyzed independently by different clinical technologists, radiologists or psychiatrists who were blinded to the data collected in other examinations.

\section{Multifractal analysis}

The idea of the multifractal is a generalized concept based on Mandelbrot's fractal (Halsey et al., 1986; Mandelbrot, 1983). Details of multifractal analysis are provided elsewhere (Takahashi et al., 2001, 2004). Briefly, after dividing an image into squares with sides having length $r$, we define a probability density for each square box as $p_{i}=N_{i} / N$, where $N_{i}$ is the density at the $i$-th box and $N=\sum_{i} N_{i}$ is the total density of the image. The density of the images with nonlinear structures fluctuates and reveals fractality. To estimate this fractality, Halsey et al. (1986) proposed the $q$-th moment of probability density to be $\sum_{i} p_{i}^{q}$, a generalization of the conventional partition function $\sum_{i} p_{i}$. When the probability scales as $p_{i} \propto r^{\alpha i}, \alpha_{i}$ represents a local fractal dimension at the $i$-th box. Halsey et al. (1986) have defined a mass index $\tau(q)$ by

$\lim _{r \rightarrow 0}\left(\sum_{i} p_{i}^{q} / r^{\tau(q)}\right)=1$.

$\tau(q)$ relates to the so-called generalized fractal dimension $D(q)$ (Grassberger, 1983; Hentschel and Procaccia, 1983) as $\tau(q)=$ $(q-1) D(q)$. By mathematical manipulation (Halsey et al., 1986), we obtain

$\alpha(q)=\mathrm{d} \tau(q) / \mathrm{d} q$.

$\alpha(q)$, the Lipschitz-Hölder index (or the strength of singularity after Halsey et al. (1986)), represents the local dimension. For the set with a constant $\alpha(q)$ on the image, Mandelbrot's fractal dimension (the capacity dimension) is given as (Halsey et al., 1986)

$f(\alpha(q))=q \alpha(q)-\tau(q)$.

Analogies between multifractal theory and classical thermodynamics can be drawn as follows: $q$ corresponds to inverse temperature, $\alpha(q)$ to energy, $f(\alpha(q))$ to entropy, and $\tau(q) / q$ to free energy.

Actually, $f(\alpha)$ is the smooth concave function of $\alpha$, typical examples of which are shown in Fig. 1. Theoretically, $\alpha(q)$ with large positive $q$ values corresponds to the most concentrated regions (low intensity regions of the image, i.e., dark regions) and $\alpha(q)$ with large negative $q$ values corresponds to the most rarefied regions (high intensity regions of the image, i.e., white regions). In addition, the width of both ends of the $f(\alpha)$ spectrum reveals the image's heterogeneity. Since $\alpha(q)$ tended to be constant at $|q|>20$ in this study, the width between the $\alpha(q)$ value at $q=-20$ (the right end of the $f(\alpha)$ spectrum in Fig. 1) and the $\alpha(q)$ value at $q=$ +20 (the left end of the $f(\alpha)$ spectrum in Fig. 1) was defined as $\Delta \alpha$ (the width of both ends of the $f(\alpha)$ spectrum in Fig. 1). Our previous studies showed that $\Delta \alpha$ is the most suitable multifractal index of heterogeneity on MRI (Takahashi et al., 2001, 2004).

Brain MRI was performed using a General Electric 1.5-T Signa system. Axial T2-weighted images $(\mathrm{TR}=3000 \mathrm{~ms}, \mathrm{TE}=$ $80 \mathrm{~ms}$ ) were obtained parallel to the orbitomeatal line. The slice 


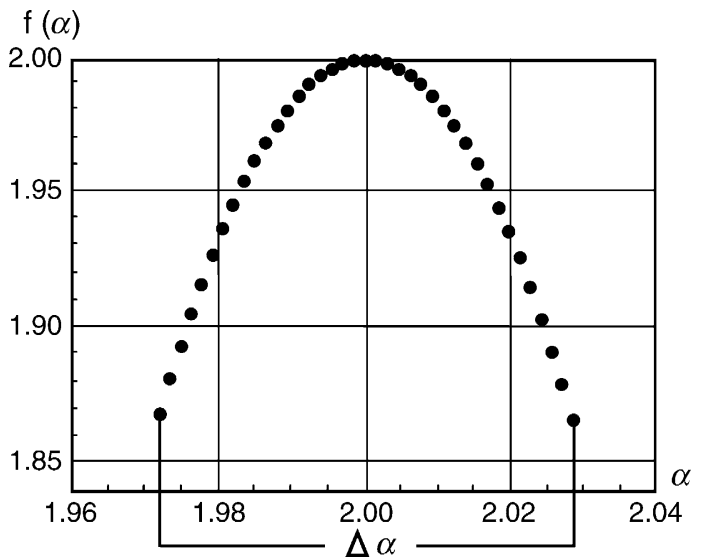

Fig. 1. Typical example of $f(\alpha)$ spectrum in a deep white matter ROI. The slope at each plot of the smooth concave function shows the $q$ value. The $\alpha(q)$ value at $q=-20$ was defined as $\alpha_{\max }$ (right end of the $f(\alpha)$ spectrum), while the $\alpha(q)$ value at $q=+20$ was defined as $\alpha_{\text {min }}$ (left end of the $f(\alpha)$ spectrum). The width of both ends of the $f(\alpha)$ spectrum was defined as $\Delta \alpha$.

thickness was $5 \mathrm{~mm}$, and the matrix size was $512 \times 512$. No spatial filter was used prior to multifractal analysis, although the Fermi spatial filter built in the GE MR imaging system was used during reconstruction. Since multifractal analysis evaluates the fluctuation of the objects, the difference of intensity range among subjects poses an adverse effect in calculating $\Delta \alpha$. Therefore, MRI signal intensity data were converted into 255gray scale images (the highest signal intensity which is most commonly distributed in background air as maximal densities and the lowest signal intensity which is most commonly distributed in cerebrospinal fluid as minimal densities) using a Scion Image Beta 3 processing application (Scion). The "power of 2" box size (i.e., $r=2^{n}$ ) is needed to divide the image to calculate the $f(\alpha)$ spectrum, and an increase in size will theoretically provide more stable results. Previously, we reported the necessity for at least $32 \times 32$ pixel images to achieve a stable $f(\alpha)$ spectrum according to the size and resolution of the images (Takahashi et al., 2004). In this study, the establishment of ROIs was restricted because we included subjects with DWMH, which must be excluded from the ROI. In the actual analysis, to achieve more robust data, a rectangular ROI of $x \times$ $y$ pixels $(1600<x \times y<2000 ; x, y>32$ pixels $)$ was placed bilaterally in the deep white matter of the frontoparietal region in the vicinity of the lateral ventricles (Figs. 2A and B) as the ROI. ROIs were chosen to avoid including the cortex, the ventricles, and the abnormal white matter intensities manually by T.T. and K.N. Since the rectangular ROI consisted of $(x-$ $32+1) \times(y-32+1)$ frames of $32 \times 32$ pixel images (Fig. $2 \mathrm{C}$ with magnification), we performed multifractal analysis to these subframes separately and averaged them all for subsequent analysis. The interrater reliability (intraclass correlation coefficient), established by two different evaluators (T.T. and K.N.), was $r=$ 0.813 for the right $\Delta \alpha$ and $r=0.912$ for the left $\Delta \alpha$. Prior to calculating the multifractal dimensions, all selected ROIs were visually inspected and determined to be normal (as shown in Figs. $3 \mathrm{E} 1$ and E2) by an experienced radiologist (H.K.). As a result of setting this condition, however, we had to exclude 7 subjects from further analysis due to restrictions in the establishment of the ROIs (confined space for setting the ROIs and high prevalence of DWMH). The MR signal intensity fluctuations in these ROIs (as shown in Fig. 2C with magnification) of the remaining subjects were quantitatively evaluated by multifractal analysis. The sum of the density of the divided image into the $i$-th square with a side of $r$ (maximum was 32 pixels) was regarded as $N_{i}$, and the sum of the density of the entire image was regarded as $N$. The probability
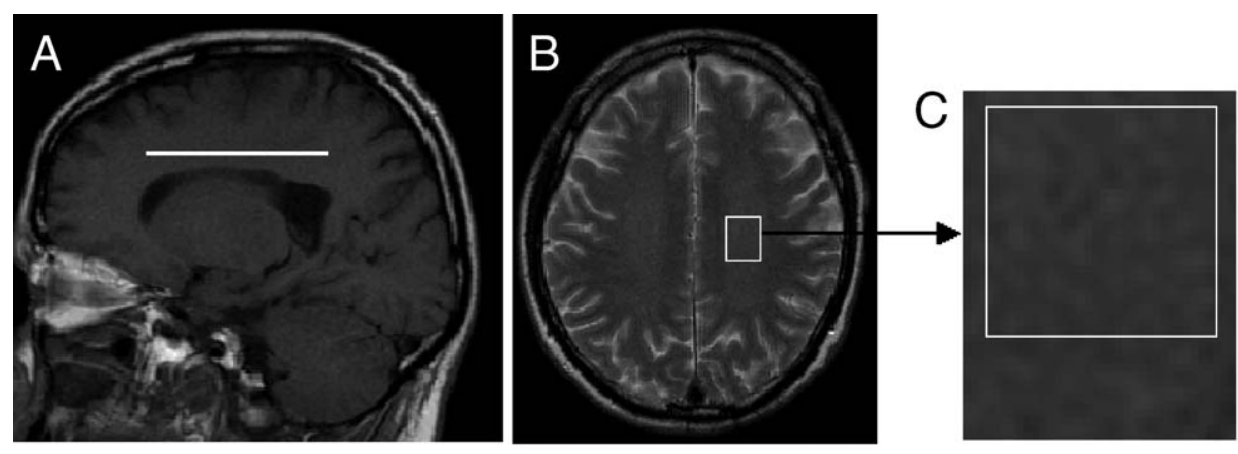

D

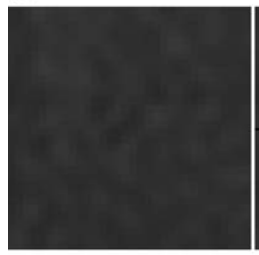

$r=32$ pixels

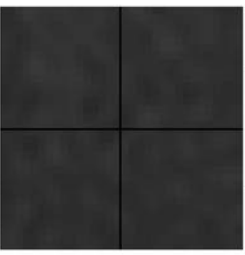

$r=16$ pixels

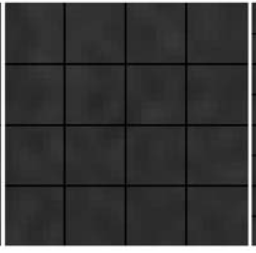

$r=8$ pixels

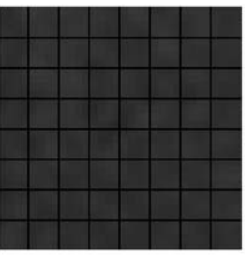

$r=4$ pixels

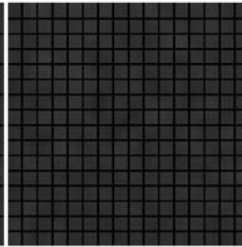

$r=2$ pixels

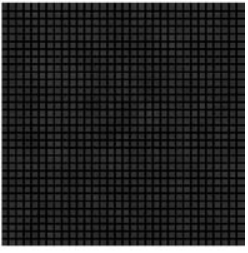

$r=1$ pixel

Fig. 2. Representative sagittal (A) and axial (B) slices from T2-weighted MR images demonstrate ROI placement. A rectangular $x \times y$ pixel ROI $(1600<x \times$ $y<2000 ; x, y>32$ pixels) was placed bilaterally in the deep white matter of the frontoparietal region in the vicinity of the lateral ventricles (white line on A and white rectangle on $\mathrm{B}$ ). ROIs were chosen manually to avoid including the cortex, the ventricles, and abnormal white matter intensities. The rectangular $x \times y$ pixel ROI consisted of $(x-32+1) \times(y-32+1)$ frames of $32 \times 32$ pixel images. A typical example of the rectangular regions of interest $($ ROIs: $38 \times 48$ pixels) on T2-weighted MR images selected from the deep white matter of the frontoparietal region (B) and MR signal intensity fluctuations in the ROI (C, with magnification). Panel D shows divided images with sides of $r=1,2,4,8,16$, and 32 pixels. 


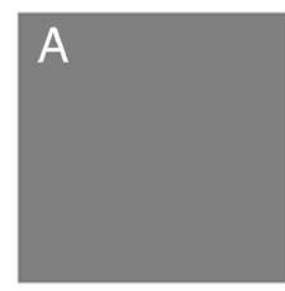

simple gray

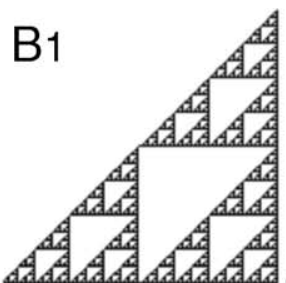

Sierpinski's gasket (homogeneous)

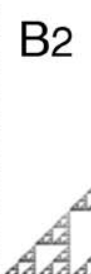

Sierpinski's gasket (heterogeneous)
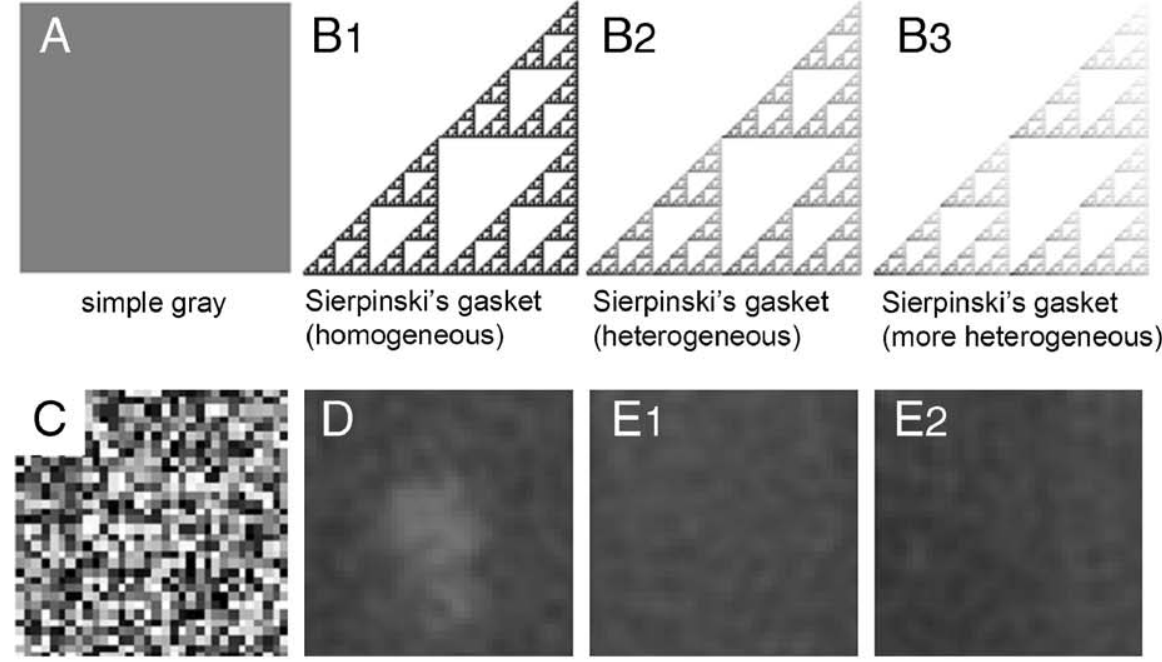

random noise

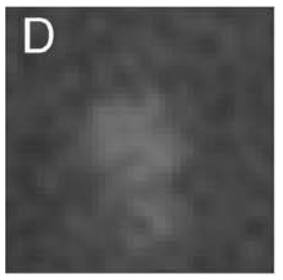

ROI with DWMH

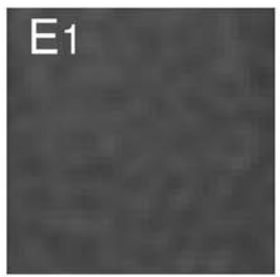

$\mathrm{ROI}$ with high $\Delta \alpha$

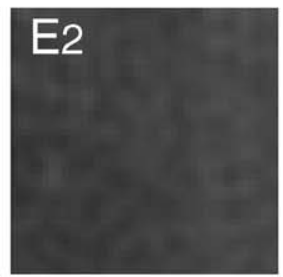

$\mathrm{ROI}$ with low $\Delta \alpha$

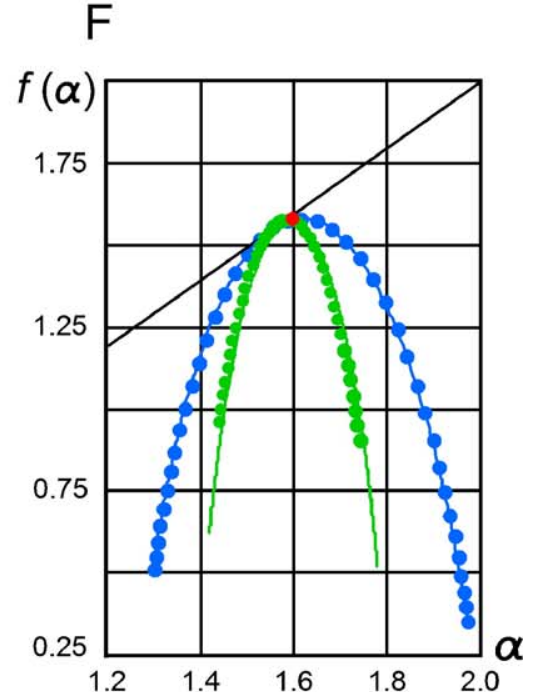

Fig. 3. Examples of simulated homogeneous images such as simple gray (A), standard fractal objects such as Sierpinski's gaskets (B1-3 from homogeneous to heterogeneous type), randomized noise (C), and averaged image of 10 ROIs with DWMH (D). Panels E1 and E2 show images of selected ROIs with high and low $\Delta \alpha$. In panel $F$, lines represent theoretical values and dots show actual values. Red dots demonstrate the theoretical and actual values of a simple Sierpinski's gasket (B1). The green line and dots show results for the heterogeneous Sierpinski's gasket (B2). The blue line and dots show the results for a more heterogeneous Sierpinski's gasket (B3). With increasing heterogeneity of the image (from B1 to B3), the width of both ends of $\alpha$ gradually increases (as shown in F).

density in the $i$-th square was defined as $p_{i}=N_{i} / N$ for 1, 2, 4, 8, 16, and 32 pixels (Fig. 2D).

DWMHs were graded to ascertain their conventional macroscopic classifications for hyperintensity on T2-weighted images according to the system of Fazekas et al. (1988) (grade $0=$ absent; grade 1 = punctuate foci; grade 2 = beginning confluence of foci; grade 3 = large confluent areas). The 36 subjects were classified as follows: grade 0 (24), 1 (10), 2 (2), or 3 (0) by a skilled radiologist (H.K.). We also divided the subjects into two groups, grade 0 and grades $1-3$, due to the small numbers of DWMHs classified specifically as grade 2 or 3 .

\section{Conventional texture analysis}

To compare multifractal analysis with conventional texture analysis, we additionally measured the SD/MSI of the absolute gradient in each ROI using the Scion Image Beta 3 processing application (Scion), in addition to calculating multifractal dimensions.

\section{Carotid B-mode ultrasonography}

Carotid B-mode ultrasonography was performed using a LOGIQ500 MD MR3 (General Electric Medical Systems), and carotid atherosclerosis was examined using an $8.8-\mathrm{MHz}$ wideband transducer. IMT was measured as the distance between the lumenintima interface and the media-adventitia interface at the far wall on each side in the B-mode image according to the method of Handa et al. (1990).

\section{Biochemical determinations}

All subjects underwent venous blood sampling from an antecubital vein in the right arm at about 11:00 a.m. after an overnight and morning fast. Serum concentrations of total cholesterol (TC) were measured by enzymatic determination.
Twenty-four-hour ambulatory blood pressure monitoring (ABPM)

Twenty-four-hour ABPM was performed using an FM-200 (Fukuda Densi Co., Tokyo, Japan). Blood pressure (BP) was measured at 30-min intervals from 6:00 a.m. to 10:00 p.m. and at one-hour intervals from 10:00 p.m. to 6:00 a.m. In this study, 24$\mathrm{h}$ mean systolic BP was used.

\section{Accuracy and sensitivity assessment of multifractal analysis}

To determine whether our method accurately measured multifractal dimensions, we simulated homogeneous images such as simple gray (Fig. 3A) and a standard fractal object such as Sierpinski's gasket (Figs. 3B1-3 from homogeneous to heterogeneous type) in comparison with theoretical values. Additionally, randomized noise (Fig. 3C), background air, and the averaged image of 10 ROIs with DWMH (Fig. 3D) were calculated. Figs. $3 \mathrm{E} 1$ and E2 show the images of selected ROIs with high and low $\Delta \alpha$. Simple gray images resulted in $\Delta \alpha$ close to 0 , which indicates that they were completely homogeneous (Fig. 3A). Delta $\alpha$ of Sierpinski's gasket was in line with the theoretical value (Fig. 3F); the line indicates the theoretical value, and the dots reveal the actual value. With increasing image fluctuation (from Figs. $3 \mathrm{~B} 1$ to B3), the width of both ends of $\alpha$ gradually increases, results in line with the algorithm of multifractals that substantiate the accuracy of our analysis. Moreover, $\Delta \alpha \mathrm{s}$ of images with DWMH were extremely large (mean: 0.156 , SD: 0.023 ) compared to those of apparently normal white matter (mean: 0.024, SD: 0.007). We also analyzed background air images in all subjects to identify the effect of background activity. We found extremely high values of $\Delta \alpha$ in background air (mean: 0.505 , SD: 0.057 , close to the value of noise) compared to those of white matter; there was no significant relationship between the $\Delta \alpha \mathrm{s}$ of background air images and those of the white matter (right side: $r=-0.052$; left side: $r=0.093$ ). These results substantiate the irrelevancy of artifacts in applying 
Table 1

Demographic characteristics of the study subjects with comparison between gender groups

\begin{tabular}{|c|c|c|c|}
\hline & Male $(n=23)$ & Female $(n=13)$ & $P$ \\
\hline $\begin{array}{l}\text { Right } \Delta \alpha, \\
\text { mean (SD) }\end{array}$ & $0.0246(0.0062)$ & $0.0237(0.0077)$ & 0.725 \\
\hline $\begin{array}{l}\text { Left } \Delta \alpha, \\
\quad \text { mean }(\mathrm{SD})\end{array}$ & $0.0251(0.0078)$ & $0.0219(0.0046)$ & 0.181 \\
\hline $\begin{array}{l}\text { Right SD/MSI, } \\
\text { mean (SD) }\end{array}$ & $0.0347(0.0071)$ & $0.0275(0.0046)$ & $0.002^{* *}$ \\
\hline $\begin{array}{r}\text { Left SD/MSI, } \\
\text { mean (SD) }\end{array}$ & $0.0340(0.0071)$ & $0.0274(0.0036)$ & $0.004 * *$ \\
\hline $\begin{array}{l}\text { Right IMT }(\mathrm{mm}), \\
\text { mean }(\mathrm{SD})\end{array}$ & $0.863(0.090)$ & $0.838(0.112)$ & 0.463 \\
\hline $\begin{array}{l}\text { Left IMT }(\mathrm{mm}), \\
\text { mean }(\mathrm{SD})\end{array}$ & $0.868(0.096)$ & $0.843(0.110)$ & 0.479 \\
\hline $\begin{array}{l}\text { Age (years), } \\
\text { mean (SD) }\end{array}$ & $60.96(4.74)$ & $60.85(4.10)$ & 0.944 \\
\hline $\begin{array}{r}\text { BMI }\left(\mathrm{kg} / \mathrm{m}^{2}\right), \\
\text { mean }(\mathrm{SD})\end{array}$ & $23.90(2.01)$ & $22.43(2.65)$ & 0.069 \\
\hline $\begin{array}{l}\mathrm{TC}(\mathrm{mg} / \mathrm{dl}), \\
\text { mean }(\mathrm{SD})\end{array}$ & 200.95 (31.59) & $227.00(37.03)$ & $0.032^{*}$ \\
\hline $\begin{array}{l}\mathrm{SBP}(\mathrm{mm} \mathrm{Hg}), \\
\text { mean }(\mathrm{SD})\end{array}$ & $126.35(13.56)$ & $119.15(11.00)$ & 0.112 \\
\hline $\begin{array}{l}\text { Co-existence of } \\
\text { DWMH }(\%),(n)\end{array}$ & $30.4(7 / 23)$ & $30.8(4 / 13)$ & 0.635 \\
\hline
\end{tabular}

Data are expressed as mean $\pm \mathrm{SD}$; SD/MSI, standard deviation of signal intensity divided by mean value of signal intensity; IMT, intima-media thickness; BMI, body mass index; TC, total cholesterol; SBP, systolic blood pressure; DWMH, deep white matter hyperintensity.

$$
* P<0.05 \text {. }
$$$$
\text { ** } P<0.01 \text {. }
$$

multifractal analysis to white matter on T2-weighted MRI. As other possible sources of error, effects of the inhomogeneities of the RF field and coil sensitivity must be taken into account for multifractal analysis. Previously, we found a significant difference in $\Delta \alpha$ between healthy young subjects and healthy elderly subjects under the same MRI conditions (Takahashi et al., 2004). This difference supports the reflection of the fluctuation of the white matter tissue itself (e.g., age-related changes in $\Delta \alpha$ ) by multifractal analysis irrespective of the inhomogeneities of the RF field and coil sensitivity.

\section{Statistical analysis}

Statistical analysis was carried out using SPSS software for Windows version 12 (SPSS Japan Inc., Tokyo, Japan).

Pearson's $\chi^{2}$ and Student's $t$ test were used to compare the distribution of categorized data and continuous data by gender. The paired $t$ test was used to test for laterality differences in $\Delta \alpha, \mathrm{SD} /$ MSI, and IMT. The relationships among $\Delta \alpha$, SD/MSI, IMT, age, gender, BMI, TC, and mean 24-h systolic BP were explored by simple correlation analysis using Pearson's product-moment correlation coefficient $(r)$. The independent IMT predictors were examined in a multiple regression model in which $\Delta \alpha, \mathrm{SD} / \mathrm{MSI}$, age, gender, and BMI were introduced as independent variables on both sides. We also calculated these univariate analysis and multiple regression models after eliminating the subjects with DWMH. The relationship between $\Delta \alpha$ and IMT was further investigated using one-way ANCOVA across IMT subgroups on both sides separately, divided into approximate groupings of high (18 subjects) and low (18 subjects) IMT values. Age, gender, and BMI were treated as covariates. $P$ values $<0.05$ were considered statistically significant.

\section{Results}

The demographic characteristics, $\Delta \alpha$, SD/MSI, IMT, age, BMI, TC, mean 24-h systolic BP, and coexistence of DWMH are presented in Table 1. There were significant differences in right and left SD/MSI and total cholesterol levels between male and female subjects.

The paired $t$ test demonstrated no significant laterality differences for $\Delta \alpha(P=0.773)$, SD/MSI $(P=0.402)$, or IMT $(P=0.625)$.

Table 2 represents the Pearson's product-moment correlation among $\Delta \alpha$, SD/MSI, IMT, age, BMI, TC, and mean 24-h systolic

Table 2

Correlation matrix of $\Delta \alpha$, SD/MSI, IMT, age, BMI, TC, and systolic BP in all subjects

\begin{tabular}{|c|c|c|c|c|c|c|}
\hline Right side & Right SD/MSI & Right IMT & Age & BMI & $\mathrm{TC}$ & SBP \\
\hline Right $\Delta \alpha$ & $0.624^{* *}$ & $0.515 * *$ & 0.109 & -0.075 & 0.101 & 0.001 \\
\hline Right SD/MSI & - & $0.397 *$ & -0.119 & $0.342 *$ & 0.232 & -0.100 \\
\hline Right IMT (mm) & - & - & 0.206 & 0.193 & -0.064 & 0.183 \\
\hline Left side & Left SD/MSI & Left IMT & Age & BMI & $\mathrm{TC}$ & SBP \\
\hline Left $\Delta \alpha$ & $0.618 * *$ & $0.474 * *$ & -0.091 & 0.106 & 0.015 & 0.127 \\
\hline Left SD/MSI & - & 0.222 & 0.163 & $0.397^{*}$ & 0.268 & -0.128 \\
\hline Left IMT (mm) & - & - & 0.324 & 0.312 & 0.051 & 0.226 \\
\hline \multicolumn{7}{|c|}{ Correlation matrix among confounding factors } \\
\hline & BMI & $\mathrm{TC}$ & SBP & & & \\
\hline Age (years) & -0.032 & 0.128 & -0.033 & & & \\
\hline BMI $\left(\mathrm{kg} / \mathrm{m}^{2}\right)$ & - & -0.066 & $0.367 *$ & & & \\
\hline $\mathrm{TC}(\mathrm{mg} / \mathrm{dl})$ & - & - & -0.121 & & & \\
\hline
\end{tabular}

SD/MSI, standard deviation of signal intensity divided by mean value of signal intensity; IMT, intima-media thickness; BMI, body mass index; TC, total cholesterol; SBP, systolic blood pressure; DWMH, deep white matter hyperintensity.

$* P<0.05$.

** $P<0.01$. 


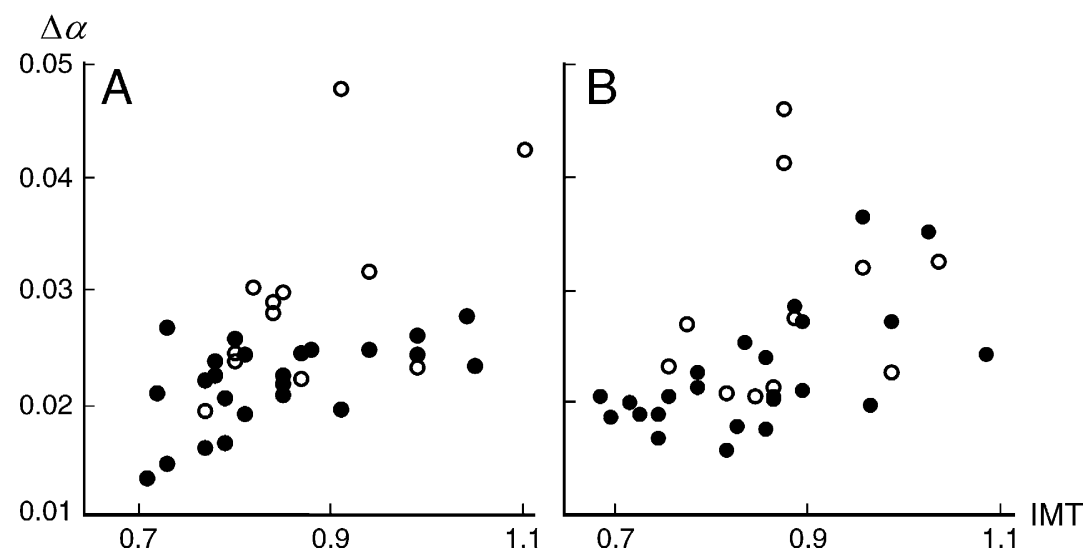

Fig. 4. Association between $\Delta \alpha$ and intima-media thickness (IMT). Significant positive correlations were observed (all subjects (open and closed circles): $r=$ $0.515, P<0.001$; subjects without DWMH (closed circles): $r=0.540, P=0.006$ ) for the right side $(\mathrm{A})$. Significant positive correlations were observed (all subjects (open and closed circles): $r=0.472, P=0.004$; subjects without DWMH (closed circles): $r=0.610, P=0.002$ ) for the left side (B).

BP in all subjects separately for right and left sides. There were significant correlations between $\Delta \alpha$ and SD/MSI for the right side $(r=0.624, P<0.001)$ and left side $(r=0.618, P<0.001)$. Correlations between texture analysis and confounding factors were found in $\Delta \alpha$ and IMT $(r=0.515, P<0.001$; open and closed circles in Fig. 4A), SD/MSI and IMT $(r=0.397, P=$ $0.016), \mathrm{SD} / \mathrm{MSI}$ and BMI $(r=0.342, P=0.041)$ for the right side, and $\Delta \alpha$ and IMT $(r=0.474, P=0.004$; open and closed circles in Fig. 4B), SD/MS and BMI ( $r=0.347, P=0.038)$ for the left side. Correlations among confounding factors were found in BMI and mean 24-h systolic BP $(r=0.367, P=0.028)$. Since there was a significant correlation between $\Delta \alpha$ and SD/MSI, we conducted multiple regression analysis with IMT for $\Delta \alpha$ and SD/ MSI. When variables $(\Delta \alpha$, age, gender, and BMI) were inputted into multiple regression models, $\Delta \alpha$ was the strongest predictor of IMT for both sides (Table 3). On the other hand, when variables (SD/MSI, age, gender, and $\mathrm{BMI}$ ) were inputted into multiple regression models, SD/MSI had no association with IMT (Table 4).

Table 5 represents the Pearson's product-moment correlation among $\Delta \alpha$, SD/MSI, IMT, age, BMI, TC, and mean 24-h systolic
$\mathrm{BP}$, in subjects without DWMH, for the right and left sides separately. There were significant correlations between $\Delta \alpha$ and SD/MSI for the right side $(r=0.592, P=0.002)$ and left side $(r=0.454, P=0.026)$. Correlations between texture analysis and confounding factors were found in $\Delta \alpha$ and IMT $(r=0.540$, $P=0.006$; closed circles in Fig. 4A), SD/MSI and BMI $(r=$ $0.453, P=0.026)$ for the right side, and $\Delta \alpha$ and IMT $(r=$ $0.610, P=0.002$; closed circles in Fig. 4B) and SD/MSI and BMI $(r=0.412, P=0.042)$ for the left side. No significant correlation was found between any combinations of confounding factors. Exclusion of subjects with DWMH did not affect the relationship between $\Delta \alpha$ and IMT in multiple regression models (Table 3).

Fig. 5 shows further evidence of the relationship between $\Delta \alpha$ and IMT. IMT was divided into approximately two subgroups (i.e., low $(<0.84 \mathrm{~mm})$, high $(\geq 0.85 \mathrm{~mm}))$, and the means across the subgroups were compared by one-way ANCOVA. One-way ANCOVA with adjustments for age, gender, and BMI indicated that the value of $\Delta \alpha$ was higher in the high-IMT group (right: $F=$ 6.942, $P=0.013$; left: $F=20.523, P<0.001)$. This significant relationship between $\Delta \alpha$ and IMT remained significant after

Table 3

Summary of multiple linear regression analysis for right and left IMT with $\Delta \alpha$

\begin{tabular}{|c|c|c|c|c|}
\hline \multirow[t]{2}{*}{ Right side } & \multicolumn{2}{|c|}{ All subjects } & \multicolumn{2}{|c|}{ Subjects without DWMH } \\
\hline & $\beta$ & $P$ & $\beta$ & $P$ \\
\hline Right $\Delta \alpha$ & 0.514 & 0.001 & 0.478 & 0.017 \\
\hline Age (years) & 0.157 & 0.293 & 0.038 & 0.828 \\
\hline Gender $(\%)$ & -0.148 & 0.883 & -0.179 & 0.355 \\
\hline BMI $\left(\mathrm{kg} / \mathrm{m}^{2}\right)$ & 0.149 & 0.145 & 0.338 & 0.071 \\
\hline \multirow[t]{2}{*}{ Left side } & \multicolumn{2}{|c|}{ All subjects } & \multicolumn{2}{|c|}{ Subjects without DWMH } \\
\hline & $\bar{\beta}$ & $P$ & $\bar{\beta}$ & $P$ \\
\hline Left $\Delta \alpha$ & 0.497 & 0.001 & 0.635 & 0.001 \\
\hline Age (years) & 0.380 & 0.008 & 0.359 & 0.025 \\
\hline Gender (\%) & 0.087 & 0.549 & 0.077 & 0.622 \\
\hline BMI $\left(\mathrm{kg} / \mathrm{m}^{2}\right)$ & 0.298 & 0.043 & 0.277 & 0.083 \\
\hline
\end{tabular}

IMT, intima-media thickness; BMI, body mass index; DWMH, deep white matter hyperintensity. $\beta$ is the standardized regression coefficient, which allows comparison of the relative contribution of each variable to the prediction of IMT. For this model, multiple $r=0.587, r^{2}=0.344, P=0.009$ (for all subjects); $r=$ $0.686, r^{2}=0.471, P=0.013$ (for subjects without DWMH) for right side and multiple $r=0.664, r^{2}=0.441, P<0.001$ (for all subjects); $r=0.779, r^{2}=0.606$, $P<0.001$ (for subjects without DWMH) for left side, respectively. 
Table 4

Summary of multiple linear regression analysis for right and left IMT with SD/MSI

\begin{tabular}{|c|c|c|c|c|}
\hline \multirow[t]{2}{*}{ Right side } & \multicolumn{2}{|c|}{ All subjects } & \multicolumn{2}{|c|}{ Subjects without DWMH } \\
\hline & $\beta$ & $P$ & $\beta$ & $P$ \\
\hline Right SD/MSI & 0.465 & 0.018 & 0.461 & 0.067 \\
\hline Age (years) & 0.265 & 0.104 & 0.122 & 0.519 \\
\hline Gender $(\%)$ & 0.131 & 0.479 & -0.161 & 0.462 \\
\hline BMI $\left(\mathrm{kg} / \mathrm{m}^{2}\right)$ & 0.082 & 0.630 & 0.105 & 0.617 \\
\hline \multirow[t]{2}{*}{ Left side } & \multicolumn{2}{|c|}{ All subjects } & \multicolumn{2}{|c|}{ Subjects without DWMH } \\
\hline & $\beta$ & $P$ & $\beta$ & $P$ \\
\hline Left SD/MSI & 0.222 & 0.239 & 0.227 & 0.363 \\
\hline Age (years) & 0.370 & 0.027 & 0.324 & 0.130 \\
\hline Gender $(\%)$ & 0.069 & 0.706 & -0.005 & 0.981 \\
\hline BMI $\left(\mathrm{kg} / \mathrm{m}^{2}\right)$ & 0.268 & 0.124 & 0.234 & 0.305 \\
\hline
\end{tabular}

SD/MSI, standard deviation of signal intensity divided by mean value of signal intensity; IMT, intima-media thickness; BMI, body mass index; DWMH, deep white matter hyperintensity. $\beta$ is the standardized regression coefficient, which allows comparison of the relative contribution of each variable to the prediction of IMT. For this model, multiple $r=0.489, r^{2}=0.239, P=0.069$ (for all subjects); $r=0.632, r^{2}=0.400, P=0.038$ (for subjects without DWMH) for right side and multiple $r=0.494, r^{2}=0.244, P=0.062$ (for all subjects); $r=0.524, r^{2}=0.274, P=0.171$ (for subjects without DWMH) for left side, respectively.

adjustments for age, gender, BMI, TC, and systolic BP (right: $F=$ 6.523, $P=0.016$; left: $F=19.092, P<0.001$ ).

\section{Discussion}

Previous studies have shown that the properties of T2-weighted MR image signal intensity reflect a broad range of pathological

Table 5

Correlation matrix of $\Delta \alpha, \mathrm{SD} / \mathrm{MSI}$, IMT, age, BMI, TC, and systolic BP in subjects without DWMH

\begin{tabular}{|c|c|c|c|c|c|c|}
\hline Right side & $\begin{array}{l}\text { Right } \\
\text { SD/MSI }\end{array}$ & $\begin{array}{l}\text { Right } \\
\text { IMT }\end{array}$ & Age & BMI & $\mathrm{TC}$ & SBP \\
\hline Right $\Delta \alpha$ & $0.592^{* *}$ & $0.540 * *$ & 0.017 & -0.024 & -0.345 & -0.071 \\
\hline $\begin{array}{l}\text { Right } \\
\text { SD/MSI }\end{array}$ & - & $0.589 * *$ & -0.064 & $0.453^{*}$ & -0.273 & 0.084 \\
\hline $\begin{array}{l}\text { Right IMT } \\
\text { (mm) }\end{array}$ & - & - & 0.128 & 0.377 & -0.215 & 0.225 \\
\hline Left side & $\begin{array}{l}\text { Left } \\
\text { SD/MSI }\end{array}$ & $\begin{array}{l}\text { Left } \\
\text { IMT }\end{array}$ & Age & BMI & $\mathrm{TC}$ & SBP \\
\hline Left $\Delta \alpha$ & $0.454 * *$ & $0.610 * *$ & -0.087 & 0.100 & 0.023 & 0.002 \\
\hline $\begin{array}{l}\text { Left } \\
\qquad \mathrm{SD} / \mathrm{MSI}\end{array}$ & - & 0.299 & -0.083 & $0.412 *$ & -0.235 & 0.011 \\
\hline $\begin{array}{l}\text { Left IMT } \\
\quad(\mathrm{mm})\end{array}$ & - & - & 0.352 & 0.392 & 0.039 & 0.226 \\
\hline
\end{tabular}

Correlation matrix among confounding factors

\begin{tabular}{llrr}
\hline & BMI & \multicolumn{1}{c}{ TC } & \multicolumn{1}{l}{ SBP } \\
\hline Age (years) & 0.197 & 0.192 & 0.127 \\
BMI $\left(\mathrm{kg} / \mathrm{m}^{2}\right)$ & - & -0.058 & 0.231 \\
TC $(\mathrm{mg} / \mathrm{dl})$ & - & - & -0.304 \\
\hline
\end{tabular}

SD/MSI, standard deviation of signal intensity divided by mean value of signal intensity; IMT, intima-media thickness; BMI, body mass index; TC, total cholesterol; SBP, systolic blood pressure; DWMH, deep white matter hyperintensity.

$$
\begin{array}{r}
* P<0.05 . \\
\text { ** } P<0.01 \text {. }
\end{array}
$$

changes, such as demyelination, microtubule deterioration, dilated perivascular spaces, and axonal dilation (Fazekas et al., 1998; Meier-Ruge et al., 1992; Takao et al., 1999). Other studies have demonstrated the roles of these changes in normal aging (Davatzikos and Resnick, 2002; Yamamoto et al., 2005), cognitive function (Gunning-Dixon and Raz, 2003; Ylikoski et al., 1993), depression (Salloway et al., 1996), and dementia (Varma et al., 2002). These pathological changes in white matter are accompanied by lesions in the small perforating cerebral arteries (Inzitari, 2003; Schmidt et al., 2002, 2004; van Swieten et al., 1991) and are related to the prevalence of peripheral atherosclerosis (Manolio et al., 1999; Pico et al., 2002). Most of these studies were based on visually detectable changes in the white matter (e.g., DWMH), occurring at relatively late stages in the development of pathological changes. These visually detectable changes could be quantified with comparative ease by visual inspection. However, to date, no studies have explored the changes that occur at the initial stages (especially in normal-appearing white matter on T2-MRI).

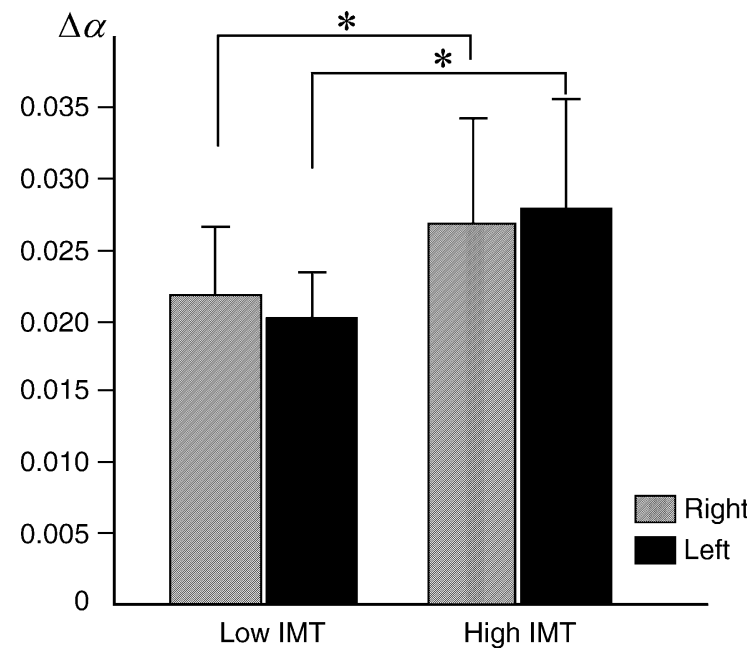

Fig. 5. $\Delta \alpha$ values across intima-media thickness (IMT) groups (high- vs. low-IMT), right and left sides, respectively. Values are mean \pm SD. $* P<0.05$ (ANCOVA). 
At the initial stages, since these MR signal intensity changes are localized in a very small region associated with cerebral small vessel damage (Inzitari, 2003; Kidwell et al., 2001; Pantoni, 2002; Pugh and Lipsitz, 2002; Schmidt et al., 2002; van Swieten et al., 1991), such minute changes might appear as fluctuations of signal intensity and be difficult to evaluate by conventional methods. Multifractal analysis can provide a good quantitative evaluation of such fluctuations in the signal intensity of the images. Our group previously reported that $\Delta \alpha$ (an index of the heterogeneity of signal intensity) in apparently normal deep white matter regions reached significantly higher levels in elderly subjects than in young subjects and was correlated with executive cognitive decline in the elderly group (Takahashi et al., 2004). However, the underlying causal mechanisms remain obscure.

In the present study, we quantitatively examined the MR signal characteristics of the deep white matter region by multifractal analysis in comparison with a conventional method of analysis, i.e., calculation of SD/MSI, and examined the correlation of these characteristics with ultrasonographic measurements of carotid artery IMT, a reliable indicator of the early stage of atherosclerosis. In univariate analysis, there was a significant correlation between $\Delta \alpha$ and SD/MSI. This correlation may be related to the fact that both parameters are evaluating some aspects of fluctuation of the signal intensity. Note that $\Delta \alpha$ was found to have a positive significant correlation with IMT in all subjects and in the subgroup of subjects without DWMH for both sides, while SD/MSI had a positive significant correlation with IMT for only the right side (Tables 2 and 5). Furthermore, we demonstrated a strong association between IMT and $\Delta \alpha$ in multiple regression models independent of age, gender, and BMI (Table 3), while there was no significant association between IMT and SD/MSI (Table 4). These results suggest that $\Delta \alpha$ more accurately reflects texture fluctuation and is a better predictor of the early stage of atherosclerosis than conventional texture analysis such as SD/MSI. More noteworthy is that the relationship between $\Delta \alpha$ and IMT was observed not only in all subjects but also after eliminating the subjects with DWMH. Thus, detection using the multifractal analysis of microstructural changes in the white matter, specifically changes related to the early stages of atherosclerosis, before the appearance of visible changes on T2-MRI, is clinically significant. IMT is a strong predictor of cerebrovascular disease, including stroke (Cao et al., 2003; Manolio et al., 1999; O'Leary et al., 1999; Touboul et al., 2000). Therefore, this study's quantitative analysis of deep white matter by multifractal analysis is likely to be useful for predicting the occurrence or prognosis of cerebrovascular disease.

Contrary to expectations, there were no significant associations between $\Delta \alpha$ or IMT and confounding factors which were reported to be associated with atherosclerosis and cerebrovascular damage (BonithonKopp et al., 1996; Bots et al., 1992; Zureik et al., 1999). In addition, very few combinations of confounding factors showed significant correlations. These results were probably related to the stringent exclusion of subjects with atherosclerotic risk factors from this study.

The findings of this study are limited by the following four factors. First, restrictions in the establishment of ROIs must be taken into account. At the present stage, multifractal analysis can only be applied to relatively large ROIs (i.e., $32 \times 32$ pixel ROIs), and ROI placement is operator-dependent. Another consideration for ROI placement is whether the pathological process affects all of the white matter or is localized. Hence, multifractal dimensions could be affected by ROI placement depending on whether the potential pathological process was included in the ROI. To overcome these limitations, we must improve multifractal analysis to permit high resolution and regional analysis. Second, artifacts derived from the MRI technique must be taken into account to enable better descriptions in multifractal analysis. Third, the subjects in the present study were highly restricted due to the exclusive selection of subjects who had no evidence of cardiovascular risk factors. Finally, the small number of subjects needs to be taken into account. Further studies with larger numbers are required to confirm our findings, and longitudinal studies are awaited to corroborate and strengthen our preliminary results. Nevertheless, our findings highlight the potential usefulness of multifractal analysis in MR imaging studies and suggest that this multifractal approach can contribute to identifying the early stages of atherosclerosis through the detection of microstructural changes in apparently normal white matter, which could be crucial in combination with conventional methods.

\section{References}

Allan, P.L., Mowbray, P.I., Lee, A.J., Fowkes, F.G., 1997. Relationship between carotid intima-media thickness and symptomatic and asymptomatic peripheral arterial disease: the Edinburgh Artery Study. Stroke $28,348-353$.

Baish, J.W., Jain, R.K., 2000. Fractals and cancer. Cancer Res. 60, $3683-3688$.

Bonithon-Kopp, C., Touboul, P.J., Berr, C., Leroux, C., Mainard, F., Courbon, D., Ducimetiere, P., 1996. Relation of intima-media thickness to atherosclerotic plaques in carotid arteries: the Vascular Aging (EVA) Study. Arterioscler. Thromb. Vasc. Biol. 16, 310-316.

Bots, M.L., Breslau, P.J., Briet, E., de Bruyn, A.M., van Vliet, H.H., van den Ouweland, F.A., de Jong, P.T., Hofman, A., Grobbee, D.E., 1992. Cardiovascular determinants of carotid artery disease: the Rotterdam Elderly Study. Hypertension 19, 717-720.

Bots, M.L., van Swieten, J.C., Breteler, M.M., de Jong, P.T., van Gijn, J., Hofman, A., Grobbee, D.E., 1993. Cerebral white matter lesions and atherosclerosis in the Rotterdam Study. Lancet 341, 1232-1237.

Burke, G.L., Evans, G.W., Riley, W.A., Sharrett, A.R., Howard, G., Barnes, R.W., Rosamond, W., Crow, R.S., Rautaharju, P.M., Heiss, G., 1995. Arterial wall thickness is associated with prevalent cardiovascular disease in middle-aged adults: the Atherosclerosis Risk in Communities (ARIC) Study. Stroke 26, 386-391.

Cao, J.J., Thach, C., Manolio, T.A., Psaty, B.M., Kuller, L.H., Chaves, P.H., Polak, J.F., Sutton-Tyrrell, K., Herrington, D.M., Price, T.R., Cushman, M., 2003. C-reactive protein, carotid intima-media thickness, and incidence of ischemic stroke in the elderly, the Cardiovascular Health Study. Circulation 108, 166-170.

Castellano, G., Bonilha, L., Li, L.M., Cendes, F., 2004. Texture analysis of medical images. Clin. Radiol. 59, 1061-1069.

Davatzikos, C., Resnick, S.M., 2002. Degenerative age changes in white matter connectivity visualized in vivo using magnetic resonance imaging. Cereb. Cortex 12, 767-771.

Fazekas, F., Niederkorn, K., Schmidt, R., Offenbacher, H., Horner, S., Bertha, G., Lechner, H., 1988. White matter signal abnormalities in normal individuals, correlation with carotid ultrasonography, cerebral blood flow measurements, and cerebrovascular risk factors. Stroke 19, 1285-1288.

Fazekas, F., Schmidt, R., Scheltens, P., 1998. Pathophysiologic mechanisms in the development of age-related white matter changes of the brain. Dement. Geriatr. Cogn. Disord. 9 (Suppl. 1), 2-5.

Grassberger, P., 1983. Generalized dimensions of strange attractors. Phys. Lett. 97A, 227-230.

Gunning-Dixon, F.M., Raz, N., 2003. Neuroanatomical correlates of selected executive functions in middle-aged and older adults: a prospective MRI study. Neuropsychologia 41, 1929-1941.

Halsey, T.C., Jensen, M.H., Kadanoff, L.P., Procaccia, I., Shraiman, B.I., 1986. Fractal measures and their singularities, the characterization of strange sets. Phys. Rev., A 33, 1141-1151. 
Handa, N., Matsumoto, M., Maeda, H., Hougaku, H., Ogawa, S., Fukunaga, R., Yoneda, S., Kimura, K., Kamada, T., 1990. Ultrasonic evaluation of early carotid atherosclerosis. Stroke 21, 1567-1572.

Hentschel, H.G.E., Procaccia, I., 1983. The infinite number of generalized dimensions of fractals and strange attractor. Physica 8D, 435-444.

Inzitari, D., 2003. Leukoaraiosis, an independent risk factor for stroke? Stroke 34, 2067-2071.

Ivanov, P.C., Amaral, L.A., Goldberger, A.L., Havlin, S., Rosenblum, M.G., Struzik, Z.R., Stanley, H.E., 1999. Multifractality in human heartbeat dynamics. Nature 399, 461-465.

Kidwell, C.S., el-Saden, S., Livshits, Z., Martin, N.A., Glenn, T.C., Saver, J.L., 2001. Transcranial Doppler pulsatility indices as a measure of diffuse small-vessel disease. J. Neuroimaging 11, 229-235.

Kirkpatrick, J.B., Hayman, L.A., 1987. White-matter lesions in MR imaging of clinically healthy brains of elderly subjects, possible pathologic basis. Radiology 162, 509-511.

Kiselev, V.G., Hahn, K.R., Auer, D.P., 2003. Is the brain cortex a fractal? NeuroImage 20, 1765-1774

Mandelbrot, B.B., 1983. The Fractal Geometry of Nature. W. H. Freeman, New York.

Manolio, T.A., Kronmal, R.A., Burke, G.L., Poirier, V., O’Leary, D.H., Gardin, J.M., Fried, L.P., Steinberg, E.P., Bryan, R.N., 1994. Magnetic resonance abnormalities and cardiovascular disease in older adults: the Cardiovascular Health Study. Stroke 25, 318-327.

Manolio, T.A., Burke, G.L., O’Leary, D.H., Evans, G., Beauchamp, N., Knepper, L., Ward, B., 1999. Relationships of cerebral MRI findings to ultrasonographic carotid atherosclerosis in older adults: the Cardiovascular Health Study. Arterioscler. Thromb. Vasc. Biol. 19, 356-365.

Mauroy, B., Filoche, M., Weibel, E.R., Sapoval, B., 2004. An optimal bronchial tree may be dangerous. Nature 427, 633-636.

Meier-Ruge, W., Ulrich, J., Bruhlmann, M., Meier, E., 1992. Age-related white matter atrophy in the human brain. Ann. N. Y. Acad. Sci. 673, $260-269$

O’Leary, D.H., Polak, J.F., Kronmal, R.A., Manolio, T.A., Burke, G.L., Wolfson, S.K. Jr., 1999. Carotid-artery intima and media thickness as a risk factor for myocardial infarction and stroke in older adults. N. Engl. J. Med. 340, 14-22.

Pantoni, L., 2002. Pathophysiology of age-related cerebral white matter changes. Cerebrovasc. Dis. 13 (Suppl. 2), 7-10.

Pico, F., Dufouil, C., Levy, C., Besancon, V., de Kersaint-Gilly, A., Bonithon-Kopp, C., Ducimetiere, P., Tzourio, C., Alperovitch, A., 2002. Longitudinal study of carotid atherosclerosis and white matter hyperintensities: the EVA-MRI cohort. Cerebrovasc. Dis. 14, 109-115.

Pugh, K.G., Lipsitz, L.A., 2002. The microvascular frontal-subcortical syndrome of aging. Neurobiol. Aging 23, 421-431.

Salloway, S., Malloy, P., Kohn, R., Gillard, E., Duffy, J., Rogg, J., Tung, G., Richardson, E., Thomas, C., Westlake, R., 1996. MRI and neuropsychological differences in early- and late-life-onset geriatric depression. Neurology 46, 1567-1574.

Schmidt, R., Fazekas, F., Enzinger, C., Ropele, S., Kapeller, P., Schmidt, H., 2002. Risk factors and progression of small vessel disease-related cerebral abnormalities. J. Neural Transm., Suppl. (62), 47-52.

Schmidt, R., Scheltens, P., Erkinjuntti, T., Pantoni, L., Markus, HS., Wallin, A., Barkhof, F., Fazekas, F., 2004. White matter lesion progression: a surrogate endpoint for trials in cerebral small-vessel disease. Neurology $63,139-144$.

Shimizu, Y., Barth, M., Windischberger, C., Moser, E., Thurner, S., 2004.
Wavelet-based multifractal analysis of fMRI time series. NeuroImage 22, $1195-1202$.

Stanley, H.E., Amaral, L.A., Goldberger, A.L., Havlin, S., Ivanov, P.Ch., Peng, C.K., 1999. Statistical physics and physiology, monofractal and multifractal approaches. Physica, A 270, 309-324.

Takahashi, T., Murata, T., Omori, M., Kimura, H., Kado, H., Kosaka, H., Takahashi, K., Itoh, H., Wada, Y., 2001. Quantitative evaluation of magnetic resonance imaging of deep white matter hyperintensity in geriatric patients by multifractal analysis. Neurosci. Lett. 314, 143-146.

Takahashi, T., Murata, T., Omori, M., Kosaka, H., Takahashi, K., Yonekura, Y., Wada, Y., 2004. Quantitative evaluation of age-related white matter microstructural changes on MRI by multifractal analysis. J. Neurol. Sci. $225,33-37$.

Takao, M., Koto, A., Tanahashi, N., Fukuuchi, Y., Takagi, M., Morinaga, S., 1999. Pathologic findings of silent hyperintense white matter lesions on MRI. J. Neurol. Sci. 167, 127-131.

Touboul, P.J., Elbaz, A., Koller, C., Lucas, C., Adrai, V., Chedru, F., Amarenco, P., 2000. Common carotid artery intima-media thickness and brain infarction: the Etude du Profil Genetique de l'Infarctus Cerebral (GENIC) case-control study. Circulation 102, 313-318.

Valenzuela, M.J., Sachdev, P.S., Wen, W., Shnier, R., Brodaty, H., Gillies, D., 2000. Dual voxel proton magnetic resonance spectroscopy in the healthy elderly, subcortical-frontal axonal $N$-acetylaspartate levels are correlated with fluid cognitive abilities independent of structural brain changes. NeuroImage 12, 747-756.

van Swieten, J.C., van den Hout, J.H., van Ketel, B.A., Hijdra, A., Wokke, J.H., van Gijn, J., 1991. Periventricular lesions in the white matter on magnetic resonance imaging in the elderly: a morphometric correlation with arteriolosclerosis and dilated perivascular spaces. Brain 114, 761-774.

Varma, A.R., Laitt, R., Lloyd, J.J., Carson, K.J., Snowden, J.S., Neary, D., Jackson, A., 2002. Diagnostic value of high signal abnormalities on T2 weighted MRI in the differentiation of Alzheimer's, frontotemporal and vascular dementias. Acta Neurol. Scand. 105, 355-364.

Yamamoto, A., Miki, Y., Tomimoto, H., Kanagaki, M., Takahashi, T., Fushimi, Y., Konishi, J., Haque, T., Togashi, K., 2005. Age-related signal intensity changes in the corpus callosum, assessment with three orthogonal FLAIR images. Eur. Radiol. 15, 2304-2311.

Ylikoski, R., Ylikoski, A., Erkinjuntti, T., Sulkava, R., Raininko, R., Tilvis, R., 1993. White matter changes in healthy elderly persons correlate with attention and speed of mental processing. Arch. Neurol. 50, 818-824.

Yoshikawa, T., Murase, K., Oku, N., Imaizumi, M., Takasawa, M., Rishu, P., Kimura, Y., Ikejiri, Y., Kitagawa, K., Hori, M., Hatazawa, J., $2003 a$. Heterogeneity of cerebral blood flow in Alzheimer disease and vascular dementia. AJNR Am. J. Neuroradiol. 24, 1341-1347.

Yoshikawa, T., Murase, K., Oku, N., Kitagawa, K., Imaizumi, M., Takasawa, M., Nishikawa, T., Matsumoto, M., Hatazawa, J., Hori, M., 2003b. Statistical image analysis of cerebral blood flow in vascular dementia with small-vessel disease. J. Nucl. Med. 44, 505-511.

Zamir, M., 1999. On fractal properties of arterial trees. J. Theor. Biol. 197, $517-526$.

Zureik, M., Touboul, P.J., Bonithon-Kopp, C., Courbon, D., Berr, C., Leroux, C., Ducimetiere, P., 1999. Cross-sectional and 4-year longitudinal associations between brachial pulse pressure and common carotid intima-media thickness in a general population: the EVA study. Stroke $30,550-555$. 